OPEN ACCESS

Edited by:

Walter Araujo Zin,

Federal University of Rio de Janeiro,

Brazil

Reviewed by: Fernanda Ferreira Cruz, Federal University of Rio de Janeiro,

Brazi

Johnatas Dutra Silva Queen's University Belfast, United Kingdom

*Correspondence:

Hsiao-Chi Chuang

r92841005@ntu.edu.tw

Specialty section: This article was submitted to Respiratory Physiology, a section of the journal

Frontiers in Physiology

Received: 14 May 2020

Accepted: 15 July 2020

Published: 12 August 2020

Citation:

Chen $X-Y$, Chen $Y-Y$, Lin W, Chien C-W, Chen C-H, Wen Y-C, Hsiao T-C and Chuang H-C (2020) Effects of Human Umbilical

Cord-Derived Mesenchymal Stem Cells on the Acute Cigarette Smoke-Induced Pulmonary

Inflammation Model.

Front. Physiol. 11:962.

doi: 10.3389/fphys.2020.00962

\section{Effects of Human Umbilical Cord-Derived Mesenchymal Stem Cells on the Acute Cigarette Smoke-Induced Pulmonary Inflammation Model}

\author{
Xiao-Yue Chen', Yi-Ying Chen', Willie Lin², Chia-Wen Chien², Chien-Han Chen², \\ Yu-Chieh Wen², Ta-Chih Hsiao ${ }^{3}$ and Hsiao-Chi Chuang ${ }^{1,4,5 *}$
}

\begin{abstract}
${ }^{1}$ School of Respiratory Therapy, College of Medicine, Taipei Medical University, Taipei, Taiwan, ${ }^{2}$ Meridigen Biotech Co. Ltd., Taipei, Taiwan, ${ }^{3}$ Graduate Institute of Environmental Engineering, National Taiwan University, Taipei, Taiwan, ${ }^{4}$ Cell Physiology and Molecular Image Research Center, Wan Fang Hospital, Taipei Medical University, Taipei, Taiwan, ${ }^{5}$ Division of Pulmonary Medicine, Department of Internal Medicine, Shuang Ho Hospital, Taipei Medical University, New Taipei City, Taiwan
\end{abstract}

Cigarette smoke (CS) has been reported to induce oxidative stress and inflammatory process in the lungs. However, the role of human umbilical cord-derived mesenchymal stem cells (hUC-MSCs) in the regulation of pulmonary inflammation remains unclear. The objective of this study is to investigate the effects of hUC-MSCs on lung inflammation in the acute CS-induced pulmonary inflammation animal model. Eightweek-old male C57BL/6 mice were intravenously administered $3 \times 10^{6}, 1 \times 10^{7}$, and $3 \times 10^{7}$ cells/kg of hUC-MSCs as well as normal saline alone (control) after 3 days of CS exposure. Mice exposed to high-efficiency particulate air (HEPA)-filtered room air served as the CS control group. High-dose $\left(3 \times 10^{7}\right.$ cells $\left./ \mathrm{kg}\right)$ hUC-MSC administration significantly decreased tumor necrosis factor (TNF)- $\alpha$ in the bronchoalveolar lavage fluid (BALF) of CS-exposed mice $(p<0.05)$. The chemokine (CXC motif) ligand 1/keratinocyte chemoattractant (CXCL1/KC) in BALF were significantly reduced by low-dose $\left(3 \times 10^{6}\right.$ cells $\left./ \mathrm{kg}\right)$ and high-dose $\left(3 \times 10^{7}\right.$ cells $\left./ \mathrm{kg}\right)$ hUC-MSC $(p<0.05)$. Medium-dose hUC-MSC administration decreased interleukin (IL)-1 $\beta$ in lung of mice, and TNF- $\alpha$ and caspase-3 were decreased in the lung of CS-exposed mice by mediumand high-dose MSC ( $p<0.05)$. Low-dose hUC-MSCs significantly elevated serum CXCL1/KC and IL-1 $\beta$ in CS-exposed mice $(p<0.05)$. Our results suggest that highdose hUC-MSCs reduced pulmonary inflammation and had antiapoptotic effects in acute pulmonary inflammation.

Keywords: cigarette smoke, inflammation, mesenchymal stem cells, oxidative stress, lung

\section{INTRODUCTION}

Cigarette smoke (CS) contains numerous toxic chemical compounds and carcinogens (Nyunoya et al., 2014). The median survival age is 85 years in never smokers, 80 years in non-daily smokers, and 75 years in daily smokers (Inoue-Choi et al., 2019). In addition, the mortality risk in non-daily smokers is $72 \%$ higher than that in never smokers. CS inhalation has been reported to activate lung epithelial cell inflammation, cause DNA damage, and induce cell death (Nyunoya et al., 2014). 
After short-term exposure to CS for 5 days, the trachea and diaphragm of mice were infiltrated by inflammatory cells (Martins et al., 2017). Long-term CS exposure increases the risks of chronic obstructive pulmonary disease, lung cancer, and premature death (Nyunoya et al., 2014). However, the treatment strategies currently used for acute pulmonary inflammation, which include bronchodilators, anti-inflammatory drugs, oxygen, and mechanical ventilation, are only supportive therapies in clinical settings (Levy and Serhan, 2014; Thompson et al., 2017; Crisafulli et al., 2018). Because of the limited number of effective therapies, a novel therapy, for example, stem cell therapy, is required to resolve acute pulmonary inflammation cases by CS exposure.

The role of mesenchymal stem cells (MSCs) in immunoregulation has been investigated for clinical purposes. A decreased level of angiopoietin-2 after the administration of a single dose of MSC has been reported in moderate to severe acute respiratory distress syndrome (Matthay et al., 2019). Following two doses of MSC and lung volume reduction surgery, cluster of differentiation (CD)-31 and $\mathrm{CD}^{+} \mathrm{T}$ cells increased in severe emphysema (Stolk et al., 2016). According to the above mentioned descriptions, MSCs have potential for lung disease treatment. However, the interaction between MSCs and lung disease should be investigated in additional studies.

MSCs are derived from the bone marrow, umbilical cord, and adipose tissue. Human umbilical cord-derived MSCs (hUCMSCs) have a more steady doubling time, rarely produce teratomas, and possess a higher differentiation capability than bone marrow-derived MSCs. Reduced expression of major histocompatibility complex classes I and II has been observed to reduce immunogenicity in hUC-MSCs (Li et al., 2015). Recently, the antifibrosis and immunomodulation properties of hUC-MSCs through prostaglandin E2 have been demonstrated (Wen et al., 2018). hUC-MSCs reduced reactive oxygen species and increased anti-inflammatory responses in acute lung injury (ALI) in vivo (Liu et al., 2016; Curley et al., 2017). hUCMSCs also mitigated inflammation and increased the percentage of regulatory $\mathrm{T}$ cells in ovalbumin-induced murine models (Kang et al., 2017).

Promoting cytokine and chemokine production can induce the homing process of MSCs in damaged tissue (Zachar et al., 2016). Most MSCs can enter the lung through intravenous infusion (Fischer et al., 2009). MSC migration to the lung may be possible due to the cell size and guidance by adhesion molecules. Therefore, MSCs have a first-pass effect on lungs because of their cellular characteristics. MSCs have been reported to attenuate lung fibrosis by decreasing pro-inflammatory cytokine production and collagen deposition ( $\mathrm{Su}$ et al., 2018). It also decreased perinatal inflammation and restored lung development in hyperoxia-induced bronchopulmonary dysplasia (Chou et al., 2016). In allergic diseases, MSCs regulated airway remodeling and reduced inflammatory cells in rhinitis and asthma mouse models (Dai et al., 2017; Isik et al., 2017). However, the effects of hUC-MSCs on CS-induced acute pulmonary inflammation still have to be elucidated. The present study investigated the effects of hUC-MSCs on lung injury and inflammation in the acute CS-induced pulmonary inflammation mouse model.

\section{MATERIALS AND METHODS}

\section{Animal}

Male C57BL/6JNarl mice (8 weeks, 20-25 g) were obtained from the National Laboratory Animal Center (Taipei, Taiwan). A constant temperature $\left(22 \pm 2^{\circ} \mathrm{C}\right)$ and relative humidity $(55 \pm 10 \%)$ and a $12 \mathrm{~h}: 12 \mathrm{~h}$ light:dark cycle were maintained throughout the study. The mice were housed in plastic cages and were administered Lab Diet 5001 (PMI Nutrition International, St. Louis, MO, United States), and they had ad libitum access to water during acclimatization. The animal experiments were performed in compliance with the Animal and Ethics Review Committee of the Laboratory Animal Center at Taipei Medical University (Taipei, Taiwan; LAC-2017-0231).

\section{Establishment of the Cigarette Smoke-Induced Acute Pulmonary Inflammation Model}

The CS exposure system had three major components, namely, a CS generator, a whole-body exposure system, and measurement devices, as illustrated in Figure 1A. The CS generator included a controller and a smoking machine for CS combustion. There were 16 slots equipped with electronic lighting control in the generator. The commercial cigarette (Mevius, Original, Japan) was continuous combustion without air suction after lighting. Clean air filtered with a high-efficiency particulate air (HEPA) filter was introduced into the CS generator. Mainstream smoke from the combustion of one commercial cigarette was then introduced into the whole-body exposure system (TECNIPLAST, Italy) at the flow rate of 15 liters per minute $(\mathrm{lpm})$ for a time course of 16 cigarettes $/ 8 \mathrm{~h} /$ day for 3 days. The mass concentration of particulate matter less than $2.5 \mu \mathrm{m}\left(\mathrm{PM}_{2.5}\right)$ was determined using a DustTrak monitor (8530, TSI, Minnesota, United States). The $\mathrm{PM}_{2.5}$ emitted from cigarette combustion was measured four times (1 time/cigarette) per day.

\section{Human Umbilical Cord-Derived Mesenchymal Stem Cell Preparation}

Human umbilical cord-derived mesenchymal stem cells were obtained from Meridigen Biotech Co., Ltd. (Taipei, Taiwan). The cells used in the present study were followed by the International Society for Cellular Therapy Guidelines. Umbilical cord tissue was harvested under sterile conditions and digested with collagenase (SERVA, Heidelberg, Germany) for $120 \mathrm{~min}$ in a $37^{\circ} \mathrm{C}$ incubator. Digestion was terminated in $\alpha$-minimal essential culture medium (Invitrogen, Waltham, MA, United States) supplemented with $18 \%$ fetal bovine serum (Invitrogen), $4 \mathrm{ng} / \mathrm{ml}$ basic fibroblast growth factor (Peprotech, Rocky Hill, NJ, United States), and $50 \mathrm{mg} / \mathrm{ml}$ gentamicin. The cells were subsequently incubated in a humidified incubator with 5\% $\mathrm{CO}_{2}$ at $37^{\circ} \mathrm{C}$ for 3 days, at which point the culture medium was replenished, and the non-adherent cells were removed. hUC-MSCs were passaged once reached $80-90 \%$ confluence to the sixth generation. For long-term storage, hUC-MSCs were suspended in CryoStor CS10 (STEMCELL Technologies, 

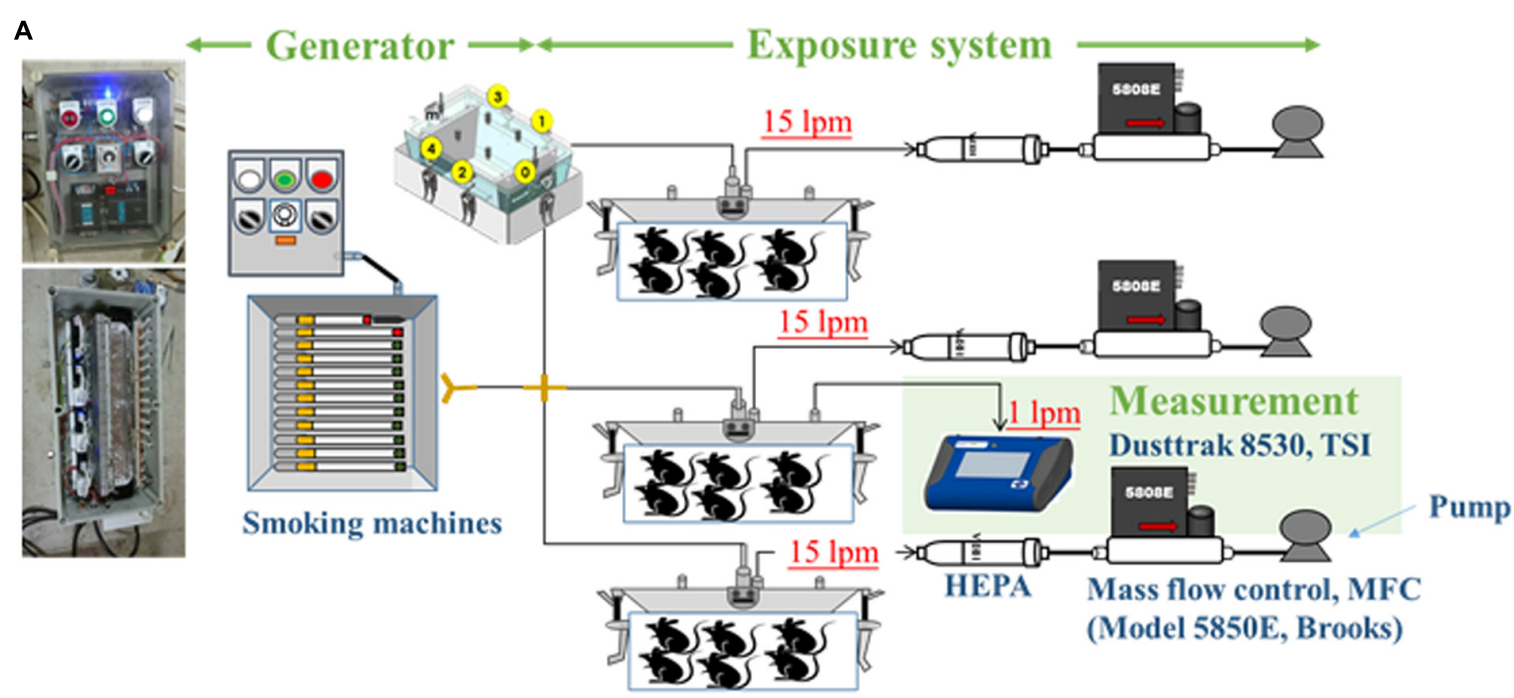

B

$\begin{array}{cc}\text { Body } & \text { Body } \\ \text { wt. } & \text { wt. }\end{array}$

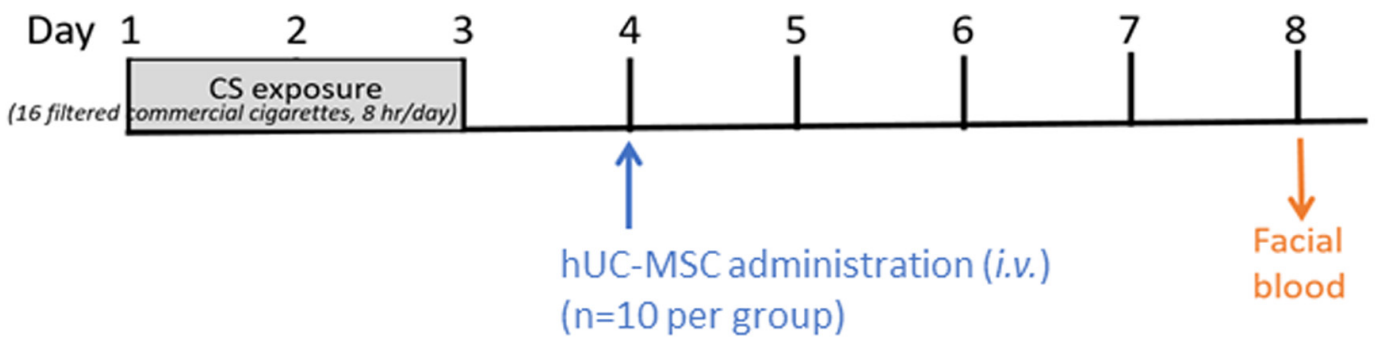

C

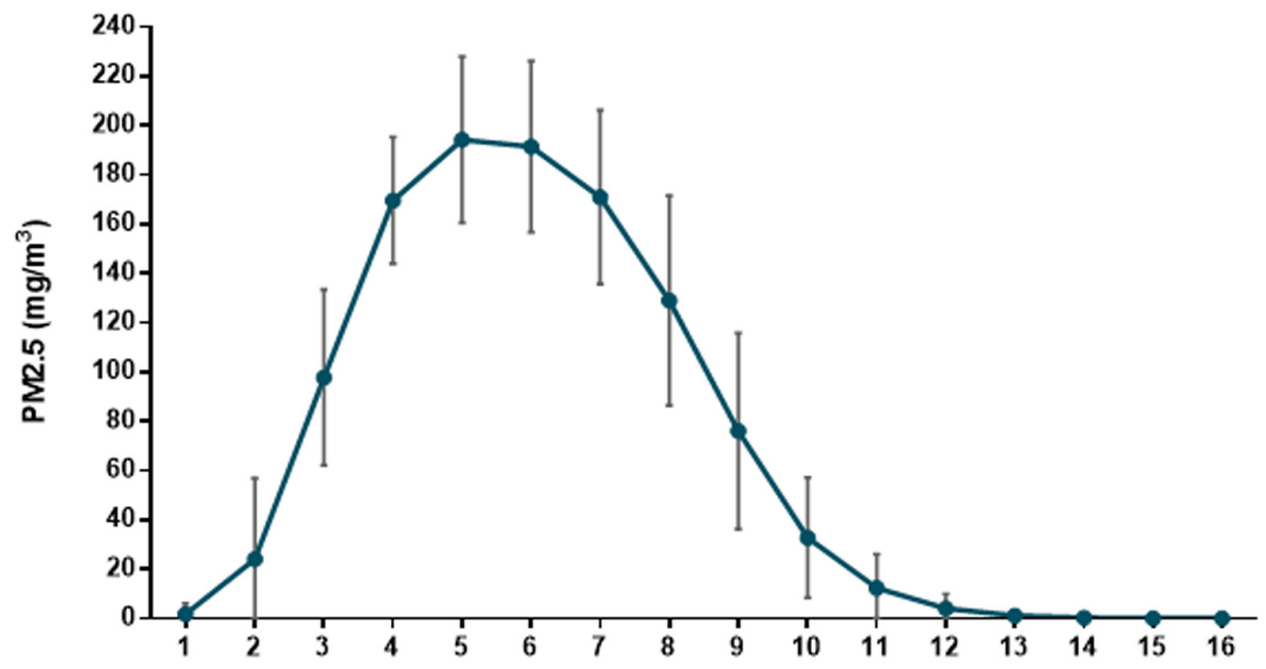

Elapsed Time ( $\mathrm{min}$ )

FIGURE 1 | Experiment design for (A) whole-body exposure system for cigarette smoke (CS), (B) human umbilical cord-derived mesenchymal stem cell (hUC-MSC) treatment on models exposed to acute CS exposure in vivo ( $n=10$ per group), and (C) distribution of PM 2.5 in mass concentrations during CS exposure. Mainstream smoke from the commercial cigarette combustion (16 cigarettes/8 h/day) was introduced into the whole-body exposure chambers. The mice in the exposure chambers were exposed to CS for 3 days. CS-exposed mice were intravenously administered a single dose of hUC-MSCs (CS + MSC-L: $3 \times 10^{6}$ cells/kg, CS + MSC-M: $1 \times 10^{7}$ cells $/ \mathrm{kg}$, and CS + MSC-H: $3 \times 10^{7}$ cells $\left./ \mathrm{kg}\right)$. All of the mice were under euthanasia on Day 8 after hUC-MSC administration. 
Vancouver, BC, Canada) and cryopreserved in a vapor phase liquid nitrogen tank. This study was approved by the Ethics Committee of the National Cheng Kung University Hospital Institutional Review Board (Tainan, Taiwan). All subjects received written and oral information prior to inclusion and provided informed consent. All study processes were carried out in accordance with the approved study protocol (A-BR-104-045).

\section{Human Umbilical Cord-Derived Mesenchymal Stem Cell Administration}

The experiment design is depicted in Figure 1B. The total number of 50 mice was used in this study and the 10 mice in each experimental group. On Day 1 , the mice were exposed to CS for 3 days. Control mice were exposed to CS-free HEPA-filtered room air (RA). On Day 4, CS-exposed mice were intravenously administered (via tail vein) a single dose of hUC-MSCs (CS + MSC-L: $3 \times 10^{6}$ cells $/ \mathrm{kg}, \mathrm{CS}+\mathrm{MSC}-\mathrm{M}: 1 \times 10^{7}$ cells $/ \mathrm{kg}$, and CS + MSC-H: $3 \times 10^{7}$ cells $/ \mathrm{kg}$ ) provided by Meridigen Biotech Co., Ltd., in clinical grade normal saline containing $2 \%$ clinical grade human serum albumin and $16.7 \%$ clinical grade CS10. Control mice were given the same volume of vehicle alone. On Day 8, all mice were anesthetized [intraperitoneally (i.p.)] under Zoletil (Virbac, Taiwan) and xylazine, followed by $\mathrm{CO}_{2}$ euthanasia. The facial blood, bronchoalveolar lavage fluid (BALF), and whole lungs were collected ( $n=10$ per group). The BALF was collected by washing the lungs three times with $0.5 \mathrm{ml}$ of phosphate-buffered saline (PBS). The BALF samples were centrifuged at $200 \mathrm{G}$ for $10 \mathrm{~min}$ at $4^{\circ} \mathrm{C}$. The supernatant and pellets were collected for biochemical and hematology analysis, respectively. The submandibular method was used for blood collection. The blood samples were stored at room temperature for an hour following centrifugation at 200G under $4^{\circ} \mathrm{C}$ for $10 \mathrm{~min}$.

\section{Body Weight}

The mouse body weight was measured on Day 1 (the day before CS exposure), Day 4 (the day before hUC-MSC administration), and Day 8.

\section{Cell Count Analysis in Bronchoalveolar Lavage Fluid}

The BALF pellets were resuspended with $40 \mu \mathrm{l}$ of PBS for hematology analysis (ProCyte Dx; IDEXX Laboratories; Westbrook, ME, United States). The total cell, neutrophil, lymphocyte, monocyte, and eosinophil counts were measured using a hematology analyzer. For the differential cell analysis, the data were presented as percentages (\%) of the total cell number.

\section{Protein Extraction in Lung Tissues}

Lung tissues were homogenized in $490 \mu \mathrm{l}$ of lysis reagent (Sigma-Aldrich, Inc., St. Louis, MO, United States) with $5 \mu \mathrm{l}$ of protease inhibitor (Geno Technology Inc., St. Louis, MO, United States) and $5 \mu \mathrm{l}$ of ethylenediaminetetraacetic acid using a homogenizer (Minilys personal homogenizer; Bertin; Rockville, MD, United States), according to the manufacturers' instructions.

\section{Enzyme-Linked Immunosorbent Assay}

Enzyme-linked immunosorbent assay (ELISA) was used to investigate the tumor necrosis factor (TNF)- $\alpha$ (Invitrogen, Waltham, MA, United States), chemokine (CXC motif) ligand 1/keratinocyte chemoattractant (CXCL1/KC; R\&D Systems Inc., Minneapolis, MN, United States), and interleukin (IL)-1 $\beta$ (Invitrogen) in the BALF and serum samples. TNF- $\alpha, \mathrm{KC}, \mathrm{IL}-1 \beta$, matrix metallopeptidase (MMP)-9 (R\&D Systems), and caspase3 levels (Elabscience, Houston, Texas, United States) in lung lysates were determined. All of the ELISA kits were conducted following the manufacturers' instructions. The levels of these markers determined in lung tissues were adjusted based on the total protein in lung lysates. The detailed information of these ELSA kits was shown in Supplementary Table S1.

\section{Statistical Analysis}

Ten mice in each group were included in each analysis. The Shapiro-Wilk's test was used to examine the normality. The data are expressed as mean \pm standard deviation (SD). The data from repeated measurement were compared using the paired $t$ test. Comparisons within multiple groups were performed through analysis of variance (ANOVA) with Fisher's post hoc test. Statistical analyses were conducted using GraphPad ver. 7 (San Diego, CA, United States) for Microsoft Windows. The significance criterion was set to $p<0.05$.

\section{RESULTS}

\section{$\mathrm{PM}_{2.5}$ Mass Concentration During Cigarette Smoke Exposure}

As presented in Figure 1C, $\mathrm{PM}_{2.5}$ was generated at an average of $108.7 \pm 72.8 \mathrm{mg} / \mathrm{m}^{3}$ during the first $10 \mathrm{~min}$ of combustion. The concentration peaked at $194.2 \pm 33.7 \mathrm{mg} / \mathrm{m}^{3}$ approximately between 5 and 6 min and started to decline afterward.

\section{Body Weight}

Figure 2A presents the body weight difference and the body weight measured on Day 8 after hUC-MSC administration. The body weight difference was determined between the mice exposed to RA, CS, and CS followed by hUC-MSC administration. There was no statistical difference between the groups.

\section{Human Umbilical Cord-Derived Mesenchymal Stem Cells Regulated Neutrophils and Monocytes in Bronchoalveolar Lavage Fluid}

The total cell counts and differential cell counts (\%) in the BALF were determined, and the results are shown in Figure 2B. There were no significant differences of total cell counts between the groups. We observed a significant increase in neutrophil (\%) production after high-dose hUC-MSC administration $\left(3 \times 10^{7}\right.$ cells $\left./ \mathrm{kg} ; p<0.05\right)$. Conversely, monocytes significantly decreased after medium- $\left(1 \times 10^{7}\right.$ cells $\left./ \mathrm{kg}\right)$, and high-dose $\left(3 \times 10^{7}\right.$ cells $\left./ \mathrm{kg}\right)$ hUC-MSC administration $(p<0.05)$. 

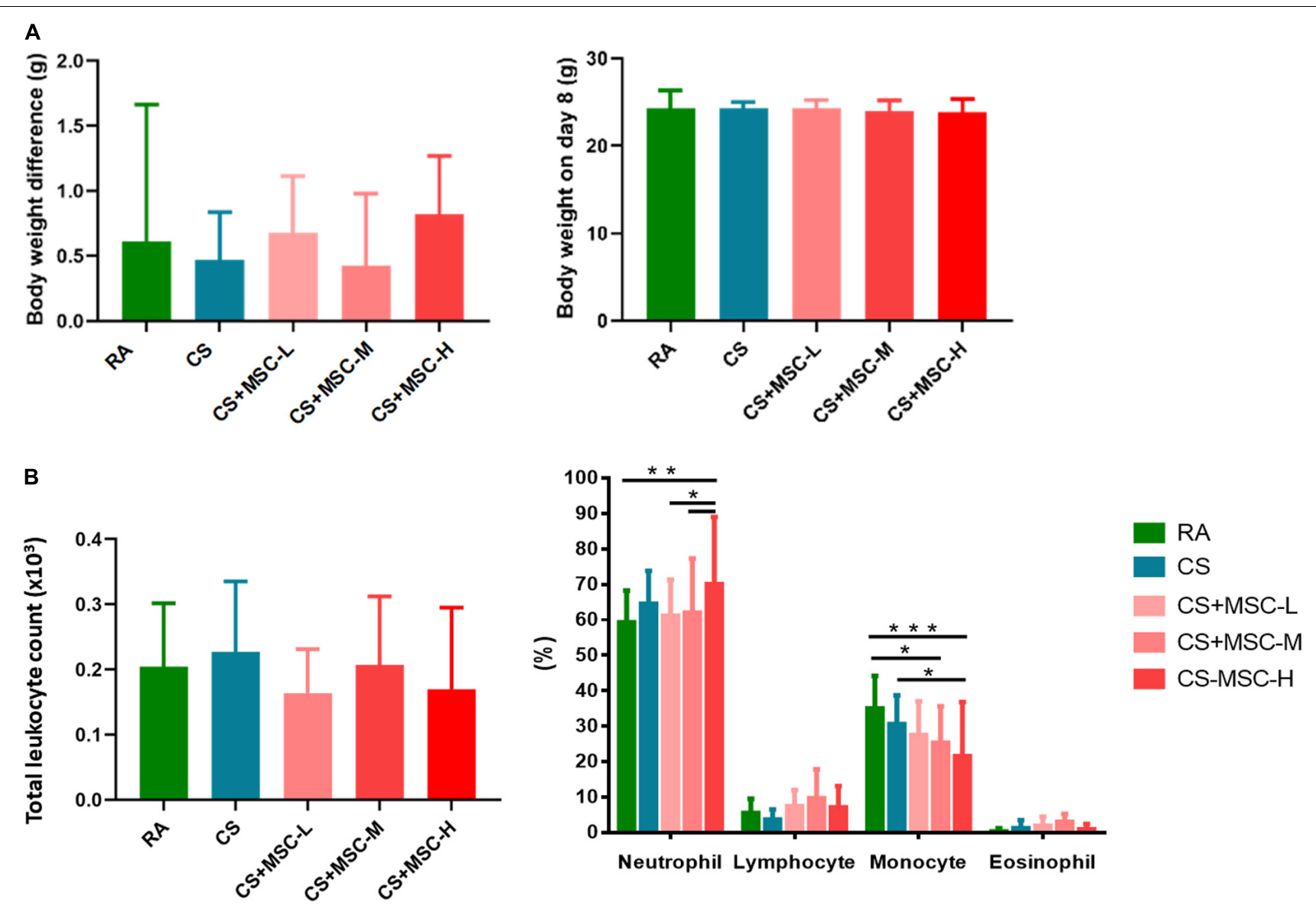

C

TNF- $\alpha$

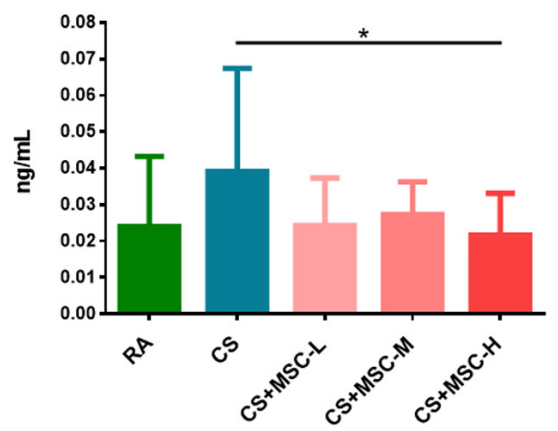

CXCL1/KC

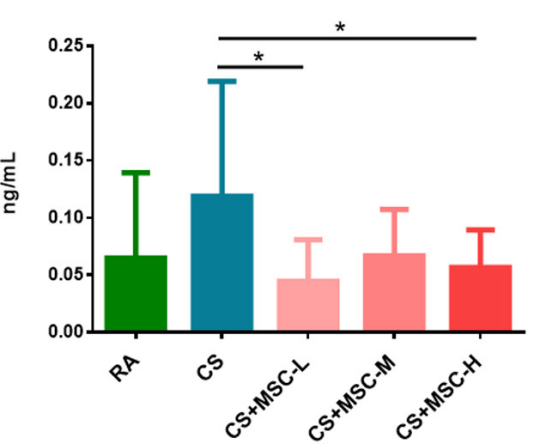

IL-1 $\beta$

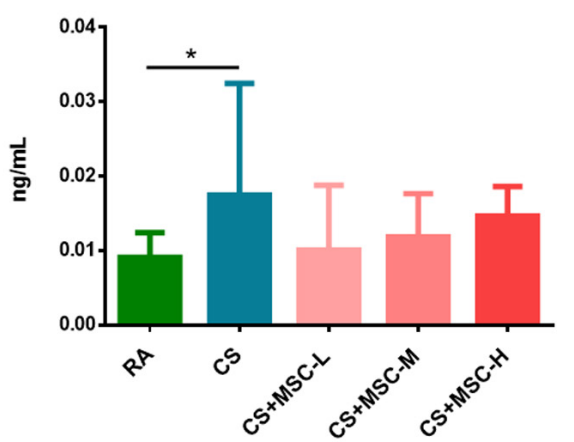

FIGURE 2 | (A) Body weight change before and after human umbilical cord-derived mesenchymal stem cell (hUC-MSC) administration; (B) Cell counts in the bronchoalveolar lavage fluid (BALF) for the room air (RA) group, cigarette smoke (CS) group, CS + MSC-L (CS with $3 \times 10^{6}$ cells/kg MSC), CS + MSC-M (CS with $1 \times 10^{7} \mathrm{cells} / \mathrm{kg} \mathrm{MSC}$ ), and CS + MSC-H (CS with $3 \times 10^{7}$ cells/kg MSC); and (C) tumor necrosis factor (TNF)- $\alpha$, (CXC motif) ligand $1 / \mathrm{keratinocyte} \mathrm{chemoattractant}$ (CXCL1/KC), and interleukin (IL)-1 $\beta$ in the BALF for the room air (RA) group, CS group, CS + MSC-L (CS with $3 \times 10^{6}$ cells/kg MSC) group, CS + MSC-M (CS with $1 \times 10^{7}$ cells $/ \mathrm{kg} \mathrm{MSC}$ ) group, and CS $+\mathrm{MSC}-\mathrm{H}$ (CS with $3 \times 10^{7}$ cells/kg MSC) group. The neutrophils were significantly increased in the CS + MSC-H group $(70.05 \pm 18.99 \%)$ than those in the RA (59.26 $\pm 8.98 \%)$, the CS + MSC-L (61.12 $\pm 10.17 \%)$, and the CS + MSC-M (62.05 $\pm 15.24 \%)$ groups. A significant decrease in monocytes (\%) was determined in the CS + MSC-H (21.52 $\pm 15.25 \%)$ when compared with those in the RA (34.98 $\pm 9.23 \%)$ and CS (30.63 $\pm 8.05 \%)$ groups. TNF- $\alpha$ was significantly decreased by CS $+\mathrm{MSC}-\mathrm{H}(0.022 \pm 0.011 \mathrm{ng} / \mathrm{ml})$ when compared with that in the CS group $(0.039 \pm 0.028 \mathrm{ng} / \mathrm{ml})$. The levels of CXCL1/KC in the CS + MSC-L $(0.045 \pm 0.036 \mathrm{ng} / \mathrm{ml})$ and CS + MSC-H $(0.056 \pm 0.033 \mathrm{ng} / \mathrm{ml})$ groups were significantly reduced compared with that in the CS group $(0.119 \pm 0.100 \mathrm{ng} / \mathrm{ml}) . n=10$ in each group. Data were presented as mean $\pm \mathrm{SD}$. The body weight difference was determined by the paired $t$ test. The cell counts and cytokines in BALF of mice were tested by ANOVA with Fisher's post hoc test. ${ }^{\star} p<0.05 ;{ }^{\star \star} p<0.01$; and ${ }^{\star \star \star} p<0.001$. 
No significant difference was observed in lymphocytes and eosinophils among the groups.

\section{Human Umbilical Cord-Derived Mesenchymal Stem Cells Decreased the Levels of Tumor Necrosis Factor- $\alpha$, (CXC Motif) Ligand 1/Keratinocyte Chemoattractant, and Interleukin-1 $\beta$ in Bronchoalveolar Lavage Fluid}

After hUC-MSC administration, TNF- $\alpha$, CXCL1/KC, and IL$1 \beta$ levels in the BALF were determined, as illustrated in Figure 2C. IL-1 $\beta$ in BALF was significantly increased in the CS group as compared with that in the RA group, indicating that the mice were induced acute lung inflammation after 3 days of CS exposure. We observed that high-dose hUC-MSC administration $\left(3 \times 10^{7}\right.$ cells $\left./ \mathrm{kg}\right)$ significantly reduced the level of TNF- $\alpha(p<0.05)$. Low- $\left(3 \times 10^{6}\right.$ cells/ $\left./ \mathrm{kg}\right)$ and high-dose $\left(3 \times 10^{7}\right.$ cells $\left./ \mathrm{kg}\right)$ hUC-MSC administration also significantly decreased CXCL1/KC $(p<0.05)$. No significant decrease in IL-1 $\beta$ was caused by hUC-MSCs after CS exposure.

\section{Human Umbilical Cord-Derived Mesenchymal Stem Cells Reduced the Levels of Tumor Necrosis Factor- $\alpha$, (CXC Motif) Ligand 1/Keratinocyte Chemoattractant, Interleukin-1 $\beta$, Matrix Metallopeptidase-9, and Caspase-3 in Lung Lysates}

The levels of TNF- $\alpha$, CXCL1/KC, IL-1 $\beta$, and MMP-9 in lung lysates were determined. As displayed in Figure 3, we observed that TNF- $\alpha$ and CXCL1/KC in lung were considerably higher in the CS group compared with those in the RA group, suggesting acute pulmonary inflammation was induced in the mice of the CS group. The TNF- $\alpha$ level was significantly reduced after medium- $\left(1 \times 10^{7}\right.$ cells/kg $)$ and high-dose $\left(3 \times 10^{7}\right.$ cells $\left./ \mathrm{kg}\right)$ hUC-MSC administration $(p<0.05)$. No significant difference was observed in CXCL1/KC production after hUC-MSC administration. Medium-dose $\left(1 \times 10^{7}\right.$ cells $\left./ \mathrm{kg}\right)$ hUC-MSC administration significantly reduced IL- $1 \beta$ in lung tissues $(p<0.05)$. There were no significant differences in MMP9 between the groups. The hUC-MSCs exhibited a significant mitigating effect on apoptosis by reducing caspase-3 $(p<0.05)$.

\section{Human Umbilical Cord-Derived Mesenchymal Stem Cells Changed the Serum Levels of Tumor Necrosis Factor- $\alpha$, (CXC Motif) Ligand $1 /$ Keratinocyte Chemoattractant, and Interleukin-1 $\beta$}

The serum levels of TNF- $\alpha$, CXCL1/KC, and IL- $1 \beta$ were determined, and the results are shown in Figure 4. TNF- $\alpha$ levels showed no significant difference among the groups. Increased CXCL1/KC levels were observed after low-dose $\left(3 \times 10^{6}\right.$ cells $\left./ \mathrm{kg}\right)$
hUC-MSC administration compared with after CS and highdose $\left(3 \times 10^{7}\right.$ cells $\left./ \mathrm{kg}\right)$ hUC-MSC administration $(p<0.05)$. The administration of low $\left(3 \times 10^{6}\right.$ cells $\left./ \mathrm{kg}\right)$ and medium $\left(1 \times 10^{7}\right.$ cells $\left./ \mathrm{kg}\right)$ doses of hUC-MSCs significantly increased IL-1 $\beta$ levels compared with RA $(p<0.05)$, whereas IL-1 $\beta$ was increased by the medium dose of hUC-MSCs when compared with that of the CS group $(p<0.05)$.

\section{DISCUSSION}

Human umbilical cord-derived mesenchymal stem cells have been reported to regulate inflammation and protect host cells from apoptosis (Li et al., 2015). In our study, we investigated the effects of hUC-MSCs on CS-induced acute pulmonary inflammation. The main results were summarized in Supplementary Table S2. We observed that hUC-MSCs mitigated pro-inflammatory cytokine production of BALF and lung tissue in the acute CS-induced pulmonary inflammation mouse model, especially at high-dose administration. hUCMSCs exerted antiapoptotic effects by reducing the caspase-3 production in the lungs of the mice. Our results suggest that high-dose of hUC-MSCs could decrease inflammatory responses during acute pulmonary inflammation in vivo.

The CS exposure system in the present study was used to determine the adverse health effects of short-term CS exposure in the lung of mice. Previous studies have reported that mice exposed to CS for 4 days showed acute lung inflammation (Nemmar et al., 2012). In the present study, mice were exposed to mainstream CS for 3 days through a whole-body exposure chamber. This exposure system and approach reduced the stress caused by CS exposure. The mice were exposed to $\mathrm{PM}_{2.5}$ at an average of $108.7 \pm 72.8 \mathrm{mg} / \mathrm{m}^{3}$ during the study period. Consistently, in previous reports, mice were exposed to CS at an average of $90-100 \mathrm{mg} / \mathrm{m}^{3}$ (Chen et al., 2005; Carter and Misra, 2010; Schweitzer et al., 2011; Siggins et al., 2014).

A previous report revealed that the majority of intravenously injected MSCs could migrate into the lungs within $24 \mathrm{~h}$ (Eggenhofer et al., 2012). Compared with bone marrow- and gingival tissue-derived MSCs, hUC-MSCs have been determined to display the highest immunomodulatory ability (Li et al., 2018). Doses of hUC-MSC between $1 \times 10^{6}$ and $4 \times 10^{6}$ cells $/ \mathrm{ml}$ have been deemed effective in a previous study (Chen et al., 2005; Carter and Misra, 2010; Lin et al., 2017). For example, the hUCMSC concentration of $3 \times 10^{6}$ cells $/ \mathrm{ml}$ was administered in lung disease (Zhang et al., 2018). However, the role of hUCMSCs in acute pulmonary inflammation has not been fully elucidated. Therefore, we investigated the effects of hUC-MSCs [intravenously (i.v.)] on the acute CS-induced lung inflammation mouse model. First, we observed body weight change after hUCMSC administration. No significant difference was found among the groups. A previous study indicated that the body weight and appetite of mice were reduced after 4 days of CS exposure (Chen et al., 2005). Another study described that the body weight of mice under chronic CS exposure increased after stem cell administration (Schweitzer et al., 2011). In the present study, the 


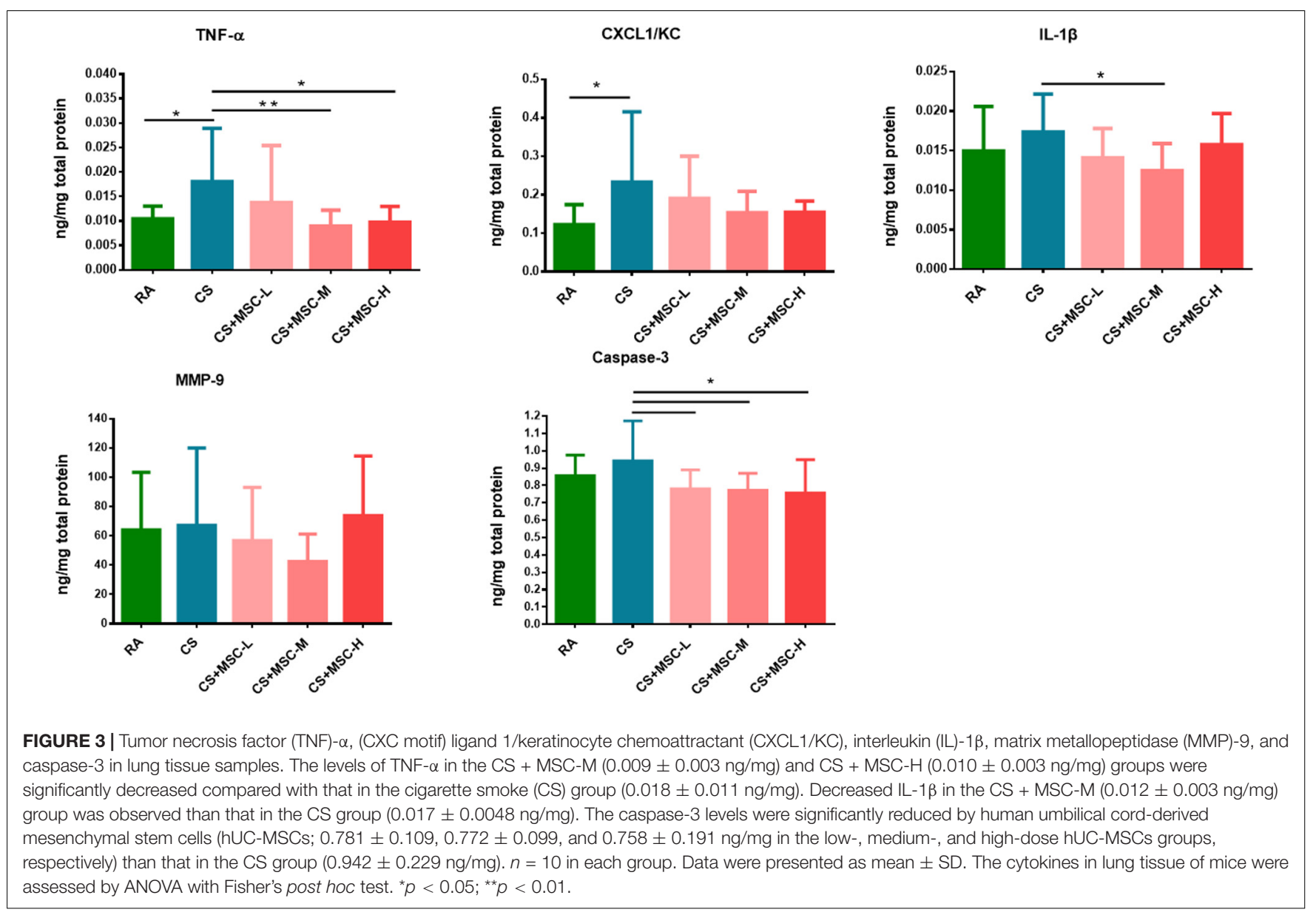

body weight slightly changed but at a non-significant statistical level, which could result from the short-term CS exposure.

In this study, we observed a significant increase in neutrophil production and a reduction of monocytes in the BALF due to hUC-MSCs. Previous studies have reported that an increase in neutrophil phagocytic activity by MSC promoted bacteria clearance in septic mouse model (Hall et al., 2013). In contrast, neutrophil counts in the BALF significantly decreased after hUC-MSC administration in Balb/c mice with lipopolysaccharide (LPS)-induced ALI (Zhu et al., 2017). MSCs decreased neutrophils, macrophages, and lymphocytes in the BALF after subacute (7 weeks) CS exposure in Sprague Dawley rats (Song et al., 2014). Both models of acute and subacute CS exposure exhibited a reduction of neutrophils in the BALF after MSC administration. The difference may be due to the various sources of MSCs as well as the tested animal models. Monocyte counts in the BALF of mice with endotoxin-induced ALI decreased after MSC administration (Hao et al., 2015). Consistently, our findings also revealed a significant reduction of monocytes in the BALF of the mice after hUC-MSC administration. The interaction between monocytes and MSCs has been reported previously. The microvesicles released by MSCs may enhance the monocyte phagocytosis and decrease inflammatory cytokine secretion (Monsel et al., 2015). Monocytes also differentiate into macrophages based on local growth factors and cytokines (Shi and Pamer, 2011). Our observation suggests that diverse regulating mechanisms of hUC-MSCs may be involved in alleviating acute pulmonary inflammation.

In this study, we observed a decrease in inflammatory TNF$\alpha$ and CXCL1/KC in the BALF by hUC-MSC, and the TNF- $\alpha$ and IL-1 $\beta$ were reduced in lung tissue of the mice after hUCMSC administration. In line with other studies, the levels of TNF- $\alpha$ and cytokine-induced neutrophil chemoattractant- 1 in the BALF of rats were reduced by MSCs at 4 and $12 \mathrm{~h}$ after gastric aspiration-induced lung injury (Zhou et al., 2016). TNF$\alpha$, IL- 6 , and macrophage inflammatory protein- 2 in the BALF decreased after MSC intervention in a rat model of ventilatorinduced lung injury (Lai et al., 2015). Most inflammatory mediators (IL-1 $\beta$, IL-6, and interferon- $\gamma$ ) in mouse lungs were mitigated within 7 days of MSC treatment in ALI (Liu et al., 2015). These observations indicate that MSCs may resolve localized pulmonary inflammation. Previous study showed that the IL-6, IL-8, and granulocyte-macrophage colony-stimulating factor (GM-CSF) secreted by MSC enhanced the migration of neutrophil to the injury site (Joel et al., 2019). Our results suggested that the increase in neutrophils and reduction of cytokines (TNF- $\alpha$, CXCL1/KC, and IL-1 $\beta$ ) in BALF and lung samples could be involved in different pathways and expressed 
TNF- $\alpha$

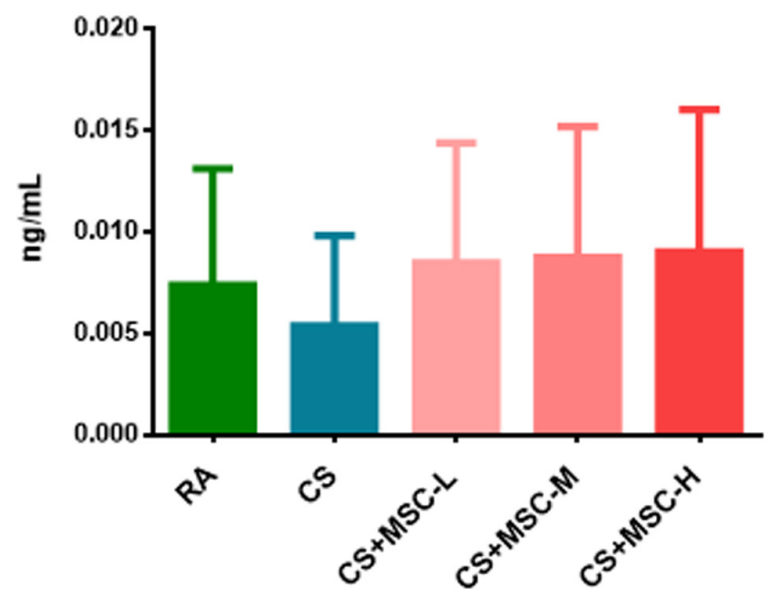

IL-1及

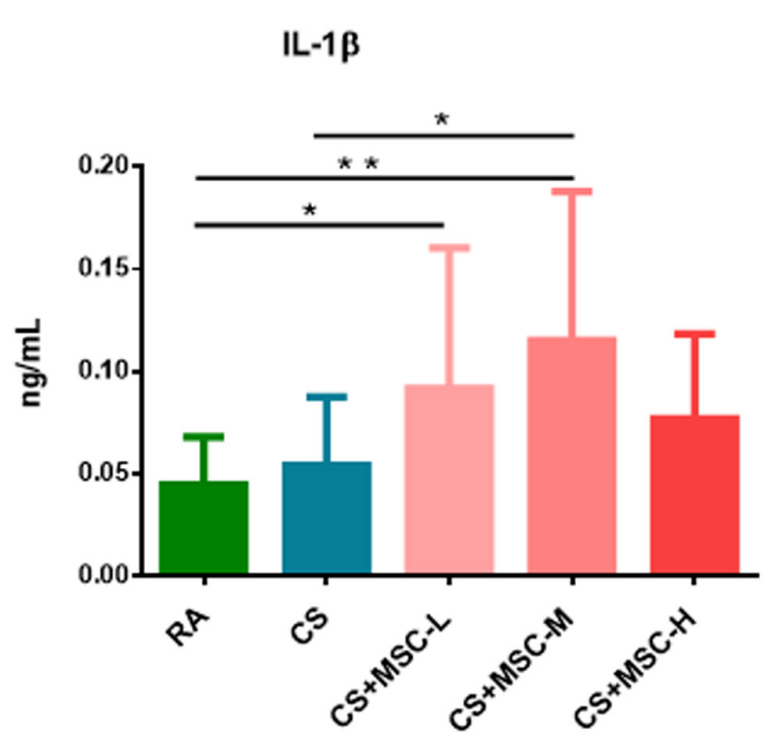

CXCL1/KC

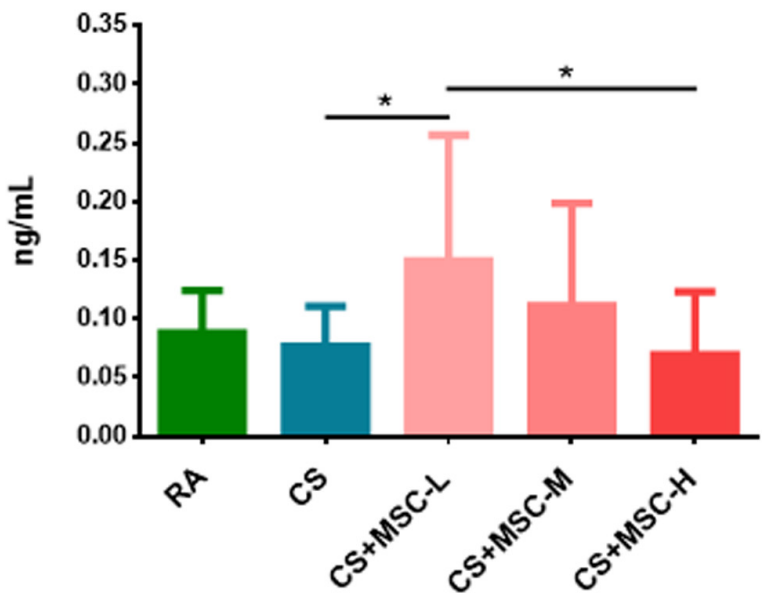

FIGURE 4 | Serum tumor necrosis factor (TNF)- $\alpha$, (CXC motif) ligand 1/keratinocyte chemoattractant (CXCL1/KC), and interleukin (IL)-1 $\beta$ changed after human umbilical cord-derived mesenchymal stem cell (hUC-MSC) administration. We observed that the level of CXCL1/KC in the CS + MSC-L group (0.150 \pm 0.107 ng/ml) was significantly increased than those in the cigarette smoke (CS; $0.076 \pm 0.034 \mathrm{ng} / \mathrm{ml})$ and CS $+\mathrm{MSC}-\mathrm{H}(0.069 \pm 0.054 \mathrm{ng} / \mathrm{ml}) \mathrm{groups}$. Increase in IL-1 $\beta$ was observed in the CS + MSC-L (0.092 $\pm 0.069 \mathrm{ng} / \mathrm{ml})$ and CS + MSC-M $(0.115 \pm 0.073 \mathrm{ng} / \mathrm{ml})$ groups when compared with that in the RA group $(0.044 \pm 0.023 \mathrm{ng} / \mathrm{ml})$, whereas IL-1 $\beta$ was significantly increased in the CS + MSC-M group $(0.115 \pm 0.073 \mathrm{ng} / \mathrm{ml})$ than that in the CS group $(0.054 \pm 0.034 \mathrm{ng} / \mathrm{ml})$. $n=10$ in each group. Data were presented as mean \pm SD. The ANOVA with Fisher's post hoc test were conducted to test the serum level of cytokines in mice. ${ }^{*} p<0.05 ;{ }^{* *} p<0.01$.

at different time points after hUC-MSC administration. Notably, in this study, we observed that the levels of caspase- 3 decreased after hUC-MSC administration. The results indicate that hUCMSCs may be involved in the regulation of apoptosis, which is also supported by previous in vivo and in vitro studies (Jiang et al., 2015; Li et al., 2017). hUC-MSCs may regulate the inflammatory responses and may exhibit antiapoptotic effects in the acute pulmonary inflammation model.

Furthermore, we discovered that the serum levels of CXCL1/KC significantly increased by low-dose human umbilical cord-derived mesenchymal stem cells. The levels of IL-1 $\beta$ in serum were increased after low and medium doses of hUCMSC administration. Intravenous infusion of MSCs in mice was proven to enhance the systemic inflammatory response [GCSF, IL-6, CXCL1, and CC motif chemokine ligand (CCL)-2] within $2 \mathrm{~h}$, which was followed by a reduction of immune reactivity (Hoogduijn et al., 2013). By contrast, previous studies have demonstrated that the MSCs significantly reduced systemic cytokines and chemokines (IL-6, IL-1b, IL-10, KC, and CCL5) after $28 \mathrm{~h}$ of administration in the sepsis-associated inflammation mouse model (Mei et al., 2010). MSCs decreased mouse IL-6 production in serum after LPS-induced acute pulmonary 
inflammation (Khedoe et al., 2017). We observed that hUCMSCs increased systemic inflammatory responses after 4 days of administration. This observation may be associated with hUCMSC-regulated neutrophil infiltration in the lungs. Neutrophils can induce IL-1 $\beta$ production (Iula et al., 2018). For example, neutrophil elastase can cleave the pro-isoform of IL-1 $\beta$ in endothelial cells to release the active form into the extracellular space (Alfaidi et al., 2015). Moreover, IL-1 $\beta$ has been shown to induce CXCL1 and CXCL2 for recruiting neutrophil infiltration in the inflammatory sites (Biondo et al., 2014). Therefore, the increase in circulating IL- $1 \beta$ and CXCL1/KC may be associated with neutrophil infiltration in the lungs of mice.

Although we determined the effects of hUC-MSCs on acute pulmonary inflammation in vivo, our study has some limitations. We observed the effects of hUC-MSCs on the acute pulmonary inflammation model; however, the regulation of hUC-MSCs in the chronic CS exposure model remains unclear. hUC-MSCs were administered for 4 days, but the chronic effects of hUCMSCs on lungs have not been determined in the present study. We noted that high-dose hUC-MSCs reduced lung inflammation but resulted in neutrophil infiltration. Additional studies are required to better understand this phenomenon. The quantity of oxidative stress and mechanistic experiments (Western blot, immunohistochemistry) will be used to investigate the role of hUC-MSC in CS-induced pulmonary inflammation in vivo. The chronic CS-induced pulmonary inflammation mouse model will be used in future works.

\section{CONCLUSION}

The present study adds to the recent research by demonstrating that hUC-MSCs mitigate pulmonary inflammation in the acute CS-induced pulmonary inflammation mouse model. Moreover, the administration of hUC-MSCs reduces apoptosis in the lungs. Our findings demonstrate that hUC-MSCs can attenuate pulmonary inflammation and may exhibit antiapoptotic effects in the short-term CS-exposed pulmonary inflammation model. hUC-MSCs may be a novel therapeutic strategy for acute pulmonary inflammation disease.

\section{DATA AVAILABILITY STATEMENT}

The raw data supporting the conclusions of this article will be made available by the authors, without undue reservation.

\section{REFERENCES}

Alfaidi, M., Wilson, H., Daigneault, M., Burnett, A., Ridger, V., Chamberlain, J., et al. (2015). Neutrophil elastase promotes interleukin-1beta secretion from human coronary endothelium. J. Biol. Chem. 290, 24067-24078. doi: 10.1074/ jbc.m115.659029

Biondo, C., Mancuso, G., Midiri, A., Signorino, G., Domina, M., Lanza Cariccio, V., et al. (2014). The interleukin-1beta/CXCL1/2/neutrophil axis mediates host protection against group B streptococcal infection. Infect. Immun. 82, 45084517. doi: 10.1128/iai.02104- 14

\section{ETHICS STATEMENT}

The studies involving human participants were reviewed and approved by Ethics Committee of the National Cheng Kung University Hospital Institutional Review Board. The patients/participants provided their written informed consent to participate in this study. The animal study was reviewed and approved by Animal and Ethics Review Committee of the Laboratory Animal Center at Taipei Medical University. Written informed consent was obtained from the owners for the participation of their animals in this study.

\section{AUTHOR CONTRIBUTIONS}

$\mathrm{H}-\mathrm{CC}$ and $\mathrm{X}-\mathrm{YC}$ contributed to the completion of interpretation of the data and the manuscript. H-CC, WL, and C-WC contributed substantially to the concept, design, interpretation of the data, and completion of the study and the manuscript. Y-YC, $\mathrm{C}-\mathrm{HC}$, and Y-CW contributed substantially to the completion of the study. T-CH contributed to the establishment of the cigarette smoke generation system and particle measurement. All authors contributed to critically revising the manuscript for important intellectual content. All authors have read and approved the final manuscript.

\section{FUNDING}

This study was supported by a grant from Meridigen Biotech Co., Ltd., Taipei, Taiwan (2017-TR-VIV-001). The fund is used for the design of the study and collection, analysis, and interpretation of data.

\section{ACKNOWLEDGMENTS}

The authors heartedly thank Miss Yi-Syuan Lin for technical assistance during this project.

\section{SUPPLEMENTARY MATERIAL}

The Supplementary Material for this article can be found online at: https://www.frontiersin.org/articles/10.3389/fphys. 2020.00962/full\#supplementary-material

Carter, C. A., and Misra, M. (2010). Effects of short-term cigarette smoke exposure on Fischer 344 rats and on selected lung proteins. Toxicol. Pathol. 38, 402-415. doi: 10.1177/0192623310364028

Chen, H., Vlahos, R., Bozinovski, S., Jones, J., Anderson, G. P., and Morris, M. J. (2005). Effect of short-term cigarette smoke exposure on body weight, appetite and brain neuropeptide Y in mice. Neuropsychopharmacology 30, 713-719. doi: 10.1038/sj.npp.1300597

Chou, H. C., Li, Y. T., and Chen, C. M. (2016). Human mesenchymal stem cells attenuate experimental bronchopulmonary dysplasia induced by perinatal inflammation and hyperoxia. Am. J. Transl. Res. 8, 342-353. 
Crisafulli, E., Barbeta, E., Ielpo, A., and Torres, A. (2018). Management of severe acute exacerbations of COPD: an updated narrative review. Multidiscip. Respir. Med. 13:36. doi: 10.1007/978-88-470-2115-0_4

Curley, G. F., Jerkic, M., Dixon, S., Hogan, G., Masterson, C., O’Toole, D., et al. (2017). Cryopreserved, xeno-free human umbilical cord mesenchymal stromal cells reduce lung injury severity and bacterial burden in rodent Escherichia coliinduced acute respiratory distress syndrome. Crit. Care Med. 45, e202-e212. doi: $10.1097 / \mathrm{ccm} .0000000000002073$

Dai, R., Liu, J., Cai, S., Zheng, C., and Zhou, X. (2017). Delivery of adipose-derived mesenchymal stem cells attenuates airway responsiveness and inflammation in a mouse model of ovalbumin-induced asthma. Am. J. Transl. Res. 9, 2421-2428.

Eggenhofer, E., Benseler, V., Kroemer, A., Popp, F. C., Geissler, E. K., Schlitt, H. J., et al. (2012). Mesenchymal stem cells are short-lived and do not migrate beyond the lungs after intravenous infusion. Front. Immunol. 3:297. doi: 10 . 3389/fimmu.2012.00297

Fischer, U. M., Harting, M. T., Jimenez, F., Monzon-Posadas, W. O., Xue, H., Savitz, S. I, et al. (2009). Pulmonary passage is a major obstacle for intravenous stem cell delivery: the pulmonary first-pass effect. Stem Cells Dev. 18, 683-692. doi: $10.1089 /$ scd.2008.0253

Hall, S. R., Tsoyi, K., Ith, B., Padera, R. F. Jr., and Lederer, J. A. (2013). Mesenchymal stromal cells improve survival during sepsis in the absence of heme oxygenase1: the importance of neutrophils. Stem Cells 31, 397-407. doi: 10.1002/stem. 1270

Hao, Q., Zhu, Y. G., Monsel, A., Gennai, S., Lee, T., Xu, F., et al. (2015). Study of bone marrow and embryonic stem cell-derived human mesenchymal stem cells for treatment of Escherichia coli endotoxin-induced acute lung injury in mice. Stem Cells Transl. Med. 4, 832-840. doi: 10.5966/sctm.20150006

Hoogduijn, M. J., Roemeling-van Rhijn, M., Engela, A. U., Korevaar, S. S., Mensah, F. K., Franquesa, M., et al. (2013). Mesenchymal stem cells induce an inflammatory response after intravenous infusion. Stem Cells Dev. 22, 2825-2835. doi: 10.1089/scd.2013.0193

Inoue-Choi, M., McNeel, T. S., Hartge, P., Caporaso, N. E., Graubard, B. I., and Freedman, N. D. (2019). Non-daily cigarette smokers: mortality risks in the u.S. Am. J. Prev. Med. 56, 27-37. doi: 10.1016/j.amepre.2018. 06.025

Isik, S., Karaman, M., Adan, A., Kiray, M., Bagriyanik, H. A., Sozmen, S. C. I, et al. (2017). Intraperitoneal mesenchymal stem cell administration ameliorates allergic rhinitis in the murine model. Eur. Arch. Otorhinolaryngol. 274, 197-207. doi: 10.1007/s00405-016-4166-3

Iula, L., Keitelman, I. A., Sabbione, F., Fuentes, F., Guzman, M., Galletti, J. G., et al. (2018). Autophagy mediates interleukin-1beta secretion in human neutrophils. Front. Immunol. 9:269. doi: 10.3389/fimmu.2018.00269

Jiang, X., Jiang, X., Qu, C., Chang, P., Zhang, C., Qu, Y., et al. (2015). Intravenous delivery of adipose-derived mesenchymal stromal cells attenuates acute radiation-induced lung injury in rats. Cytotherapy 17, 560-570. doi: 10.1016/j.jcyt.2015.02.011

Joel, M. D. M., Yuan, J., Wang, J., Yan, Y., Qian, H., Zhang, X., et al. (2019). MSC: immunoregulatory effects, roles on neutrophils and evolving clinical potentials. Am. J. Transl. Res. 11, 3890-3904.

Kang, S. Y., Park, D. E., Song, W. J., Bae, B. R., Lee, J. W., Sohn, K. H., et al. (2017). Immunologic regulatory effects of human umbilical cord blood-derived mesenchymal stem cells in a murine ovalbumin asthma model. Clin. Exp. Allergy 47, 937-945. doi: 10.1111/cea.12920

Khedoe, P., de Kleijn, S., van Oeveren-Rietdijk, A. M., Plomp, J. J., de Boer, H. C., van Pel, M., et al. (2017). Acute and chronic effects of treatment with mesenchymal stromal cells on LPS-induced pulmonary inflammation, emphysema and atherosclerosis development. PLoS One 12:e0183741. doi: 10. 1371/journal.pone.0183741

Lai, T. S., Wang, Z. H., and Cai, S. X. (2015). Mesenchymal stem cell attenuates neutrophil-predominant inflammation and acute lung injury in an in vivo rat model of ventilator-induced lung injury. Chin. Med. J. 128, 361-367. doi: 10.4103/0366-6999.150106

Levy, B. D., and Serhan, C. N. (2014). Resolution of acute inflammation in the lung. Annu. Rev. Physiol. 76, 467-492.

Li, J., Xu, S. Q., Zhao, Y. M., Yu, S., Ge, L. H., and Xu, B. H. (2018). Comparison of the biological characteristics of human mesenchymal stem cells derived from exfoliated deciduous teeth, bone marrow, gingival tissue, and umbilical cord. Mol. Med. Rep. 18, 4969-4977.

Li, T., Xia, M., Gao, Y., Chen, Y., and Xu, Y. (2015). Human umbilical cord mesenchymal stem cells: an overview of their potential in cell-based therapy. Expert Opin. Biol. Ther. 15, 1293-1306. doi: 10.1517/14712598.2015.1051528

Li, X., Zhang, Y., Liang, Y., Cui, Y., Yeung, S. C., Ip, M. S., et al. (2017). iPSCderived mesenchymal stem cells exert SCF-dependent recovery of cigarette smoke-induced apoptosis/proliferation imbalance in airway cells. J. Cell Mol. Med. 21, 265-277. doi: 10.1111/jcmm. 12962

Lin, W., Hsuan, Y. C., Lin, M. T., Kuo, T. W., Lin, C. H., Su, Y. C., et al. (2017). Human umbilical cord mesenchymal stem cells preserve adult newborn neurons and reduce neurological injury after cerebral ischemia by reducing the number of hypertrophic microglia/macrophages. Cell Transplant 26, 17981810. doi: $10.1177 / 0963689717728936$

Liu, F. B., Lin, Q., and Liu, Z. W. (2016). A study on the role of apoptotic human umbilical cord mesenchymal stem cells in bleomycin-induced acute lung injury in rat models. Eur. Rev. Med. Pharmacol. Sci. 20, 969-982.

Liu, L., He, H., Liu, A., Xu, J., Han, J., Chen, Q., et al. (2015). Therapeutic effects of bone marrow-derived mesenchymal stem cells in models of pulmonary and extrapulmonary acute lung injury. Cell Transplant 24, 2629-2642. doi: 10.3727/ $096368915 \times 687499$

Martins, T. L., Campos, K. K. D., Araujo, N., Machado, D. F., and Bezerra, F. S. (2017). Extrapulmonary effects of temporal exposure to cigarette smoke. Toxicol. Ind. Health 33, 717-725. doi: 10.1177/0748233717715187

Matthay, M. A., Calfee, C. S., Zhuo, H., Thompson, B. T., Wilson, J. G., Levitt, J. E., et al. (2019). Treatment with allogeneic mesenchymal stromal cells for moderate to severe acute respiratory distress syndrome (START study): a randomised phase 2a safety trial. Lancet Respir. Med. 7, 154-162. doi: 10.1016/s22132600(18)30418-1

Mei, S. H., Haitsma, J. J., Dos Santos, C. C., Deng, Y., Lai, P. F., Slutsky, A. S., et al. (2010). Mesenchymal stem cells reduce inflammation while enhancing bacterial clearance and improving survival in sepsis. Am. J. Respir. Crit. Care Med. 182, 1047-1057. doi: 10.1164/rccm.201001-0010oc

Monsel, A., Zhu, Y. G., Gennai, S., Hao, Q., Hu, S., Rouby, J. J., et al. (2015). Therapeutic effects of human mesenchymal stem cell-derived microvesicles in severe pneumonia in mice. Am. J. Respir. Crit. Care Med. 192, 324-336. doi: $10.1164 / \mathrm{rccm} .201410-1765$ oc

Nemmar, A., Raza, H., Subramaniyan, D., John, A., Elwasila, M., Ali, B. H., et al. (2012). Evaluation of the pulmonary effects of short-term nose-only cigarette smoke exposure in mice. Exp. Biol. Med. 237, 1449-1456. doi: 10.1258/ebm. 2012.012103

Nyunoya, T., Mebratu, Y., Contreras, A., Delgado, M., Chand, H. S., and Tesfaigzi, Y. (2014). Molecular processes that drive cigarette smoke-induced epithelial cell fate of the lung. Am. J. Respir. Cell Mol. Biol. 50, 471-482. doi: 10.1165/rcmb. 2013-0348tr

Schweitzer, K. S., Johnstone, B. H., Garrison, J., Rush, N. I., Cooper, S., Traktuev, D. O., et al. (2011). Adipose stem cell treatment in mice attenuates lung and systemic injury induced by cigarette smoking. Am. J. Respir. Crit. Care Med. 183, 215-225. doi: 10.1164/rccm.201001-0126oc

Shi, C., and Pamer, E. G. (2011). Monocyte recruitment during infection and inflammation. Nat. Rev. Immunol. 11, 762-774. doi: 10.1038/nri3070

Siggins, R. W., Hossain, F., Rehman, T., Melvan, J. N., Zhang, P., and Welsh, D. A. (2014). Cigarette smoke alters the hematopoietic stem cell niche. Med. Sci. 2, 37-50. doi: 10.3390/medsci2010037

Song, L., Guan, X. J., Chen, X., Cui, Z. L., Han, F. F., Guo, X. J., et al. (2014). Mesenchymal stem cells reduce cigarette smoke-induced inflammation and airflow obstruction in rats via TGF-betal signaling. COPD 11, 582-590. doi: $10.3109 / 15412555.2014 .898032$

Stolk, J., Broekman, W., Mauad, T., Zwaginga, J. J., Roelofs, H., Fibbe, W. E., et al. (2016). A phase I study for intravenous autologous mesenchymal stromal cell administration to patients with severe emphysema. QJM 109, 331-336. doi: 10.1093/qjmed/hcw001

Su, X., Yang, L., Yin, Y., Huang, J., Qiao, F., Fang, Y., et al. (2018). Bone marrow mesenchymal stem cells tune the differentiation of myeloid-derived suppressor cells in bleomycin-induced lung injury. Stem Cell Res. Ther. 9:253.

Thompson, B. T., Chambers, R. C., and Liu, K. D. (2017). Acute respiratory distress syndrome. N. Engl. J. Med. 377, 562-572. 
Wen, Y. C., Du, M. K., Li, M. W., Hsuan, Y. C., Su, Y. C., and Lin, W. (2018). EphA2-positive human umbilical cord-derived mesenchymal stem cells exert anti-fibrosis and immunomodulatory activities via secretion of prostaglandin E2. Taiwan J. Obstet. Gynecol. 57, 722-725. doi: 10.1016/j.tjog.2018.08.020

Zachar, L., Bacenkova, D., and Rosocha, J. (2016). Activation, homing, and role of the mesenchymal stem cells in the inflammatory environment. J. Inflamm. Res. 9, 231-240. doi: 10.2147/jir.s121994

Zhang, L. C., Wang, Y., Liu, W., Zhang, X. M., Fan, M., and Zhao, M. (2018). Protective effects of SOD2 overexpression in human umbilical cord mesenchymal stem cells on lung injury induced by acute paraquat poisoning in rats. Life Sci. 214, 11-21. doi: 10.1016/j.lfs.2018. 10.020

Zhou, J., Jiang, L., Long, X., Fu, C., Wang, X., Wu, X., et al. (2016). Bone-marrowderived mesenchymal stem cells inhibit gastric aspiration lung injury and inflammation in rats. J. Cell Mol. Med. 20, 1706-1717. doi: 10.1111/jcmm.12866
Zhu, H., Xiong, Y., Xia, Y., Zhang, R., Tian, D., Wang, T., et al. (2017). Therapeutic effects of human umbilical cord-derived mesenchymal stem cells in acute lung injury mice. Sci. Rep. 7:39889.

Conflict of Interest: The authors declare that the research was conducted in the absence of any commercial or financial relationships that could be construed as a potential conflict of interest.

Copyright $\odot 2020$ Chen, Chen, Lin, Chien, Chen, Wen, Hsiao and Chuang. This is an open-access article distributed under the terms of the Creative Commons Attribution License (CC BY). The use, distribution or reproduction in other forums is permitted, provided the original author(s) and the copyright owner(s) are credited and that the original publication in this journal is cited, in accordance with accepted academic practice. No use, distribution or reproduction is permitted which does not comply with these terms. 